\title{
Spectral characterization and Schrödinger operator of space-like submanifolds
}

\author{
Shichang Shu and Tianmin Zhu
}

\begin{abstract}
In this paper, we would like to study space-like submanifolds in a de Sitter spaces $S_{p}^{n+p}(1)$. We define and discuss three Schrödinger operators $L_{H}, L_{R}, L_{R / H}$ and obtain some spectral characterizations of totally umbilical space-like submanifolds in terms of the first eigenvalue of the Schrödinger operators $L_{H}, L_{R}$ and $L_{R / H}$ respectively.
\end{abstract}

\section{Introduction}

Let $M_{p}^{n+p}(c)$ be an $(n+p)$-dimensional connected semi-Riemannian manifold of constant curvature $c$ whose index is $p$. It is called an indefinite space form of index $p$ and simply a space form when $p=0$. If $c>0$, we call it a de Sitter space of index $p$, and denote it by $S_{p}^{n+p}(c)$. A submanifold in a de Sitter space is said to be space-like if the induced metric on the submanifold is positive definite. It was pointed out by Marsden and Tipler [11] and Stumbles [16] that space-like hypersurfaces with constant mean curvature in arbitrary space-time get interested in the relativity theory. Therefore, space-like hypersurfaces in a de Sitter space have recently been investigated by many mathematicians from both physics and mathematical points of view, see for instance $[1,4,10,12]$.

We know that hypersurfaces with constant mean curvature in a Riemannian manifold $M^{n+1}(c)$ of constant sectional curvature $c$ are critical points of

Key Words: Space-like submanifold, Schrödinger operator, mean curvature, scalar curvature, first eigenvalue.

2010 Mathematics Subject Classification: Primary: 53C42; Secondary: 53A10.

Received: November, 2012.

Revised: September, 2014.

Accepted: September, 2014. 
the area functional under variations that keep constant a certain volume function. Barbosa, do Carmo and Eschenburg [3] studied the stability for hypersurfaces of constant mean curvature in Riemannian manifolds. In analogy with the case of constant mean curvature, questions of stability can be considered for hypersurfaces with constant scalar curvature. In [2], Alencar, do Carmo and Colares extended the study of stability to hypersurfaces with constant scalar curvature. As researched in $\mathrm{C}$. Wu [17] for minimal submanifolds in a unit sphere, A.A. Barros et al. [6] and Cheng [8] studied the first eigenvalues of some Schrödinger operators of submanifolds with parallel mean curvature vector or hypersurfaces with constant scalar curvature in a unit sphere and obtained some spectral characterizations of so called Veronese surface, Clifford torus or Riemannian product $S^{m}(r) \times S^{n-m}\left(\sqrt{1-r^{2}}\right), 1 \leq m \leq n-1$.

In connection with the stability for hypersurfaces with constant mean curvature or constant scalar curvature in Riemannian manifolds, Barbosa-Oliker [4] and Liu-Deng [10] studied the stability for space-like hypersurfaces with constant mean curvature or constant scalar curvature in Lorentz manifolds. From Barbosa-Oliker [4] and [5], we know that constant mean curvature spacelike hypersurfaces are solutions to a variational problem. In fact, they are critical points of the area functional for variations that leave constant a certain volume function.

In the present paper, we would like to study space-like submanifolds in a de Sitter spaces $S_{p}^{n+p}(1)$. We will define and discuss three Schrödinger operators $L_{H}, L_{R}, L_{R / H}$ and obtain some spectral characterizations of totally umbilical space-like submanifolds in terms of the first eigenvalue of the Schrödinger operators $L_{H}, L_{R}$ and $L_{R / H}$ respectively.

Take an immersion $\psi: M^{n} \rightarrow S_{p}^{n+p}(1)$ and choose a suitable pseudoorthonormal frame field $\left\{e_{1}, \ldots, e_{n}, e_{n+1}, \ldots, e_{n+p}\right\}$ adapted to the immersion $\psi$ and its associated coframe $\left\{\omega_{1}, \ldots, \omega_{n}, \omega_{n+1}, \ldots, \omega_{n+p}\right\}$. Now recall the symmetric traceless tensor introduced by Cheng and Yau in [9], which may be given by $\phi=\sum_{i, j, \alpha} \phi_{i j}^{\alpha} \omega_{i} \otimes \omega_{j} \otimes \omega_{\alpha}$, where $i, j=1, \ldots, n, \alpha=n+$ $1, \ldots, n+p, \phi_{i j}^{\alpha}=h_{i j}^{\alpha}-\frac{1}{n} \operatorname{tr} H^{\alpha} \delta_{i j}, H^{\alpha}=\left(h_{i j}^{\alpha}\right)$ and $h_{i j}^{\alpha}$ are the coefficients of the second fundamental form in the direction $e_{\alpha}$. It is easy to verify that $|\phi|^{2}=\sum_{i, j, \alpha}\left(\phi_{i j}^{\alpha}\right)^{2}=|h|^{2}-n H^{2}$, where $|h|^{2}$ denotes the squared norm of the second fundamental form of $M^{n}$. We know that $|\phi|^{2} \equiv 0$ if and only if $M^{n}$ is totally umbilical.

Before announcing our main results, we introduce three Schrödinger operators:

$$
\begin{gathered}
L_{H}=-\Delta+\frac{1}{p}|\phi|^{2}-\frac{n(n-2)}{\sqrt{n(n-1)}} H|\phi|, \\
L_{R}=-\square+\frac{1}{n p H}|\phi|^{4}+\frac{1-H^{2}}{H}|\phi|^{2},
\end{gathered}
$$




$$
L_{R / H}=-L+\frac{1}{n p H}|\phi|^{4}+\frac{1-H^{2}}{H}|\phi|^{2},
$$

where $\Delta$ is the Laplace-Beltrami operator, $\square$ is the differential operator $\square f=$ $\sum_{i, j=1}^{n}\left(n H \delta_{i j}-h_{i j}^{n+1}\right) f_{i j}$ and $L=\square+(k / 2 n) \Delta$ is a differential operator defined by the linear combination of $\Delta$ and $\square$.

Now we state the spectral characterizations of totally umbilical space-like submanifolds in terms of the first eigenvalue of the Schrödinger operators $L_{H}$, $L_{R}$ and $L_{R / H}$ as follows:

Theorem 1.1. Let $M^{n}$ be an $n$-dimensional compact orientable spacelike submanifold in an $(n+p)$-dimensional de Sitter space $S_{p}^{n+p}(1)$ with nonzero parallel mean curvature vector field. Denote by $\lambda_{1}^{L_{H}}$ the first eigenvalue of the Schrödinger operator $L_{H}$. If $\lambda_{1}^{L_{H}} \geq-n\left(1-H^{2}\right)$, then $M^{n}$ is totally umbilical.

Theorem 1.2. $\quad$ Let $M^{n}$ be an $n$-dimensional compact orientable space-like submanifold in an $(n+p)$-dimensional de Sitter space $S_{p}^{n+p}(1)$ with constant scalar curvature $n(n-1) R, R<1$ and parallel normalized mean curvature vector field. Denote by $\lambda_{1}^{L_{R}}$ the first eigenvalue of the Schrödinger operator $L_{R}$. Then $\lambda_{1}^{L_{R}} \leq \frac{n-2}{\sqrt{n(n-1)}} \max |\phi|^{3}$, and $\lambda_{1}^{L_{R}}=\frac{n-2}{\sqrt{n(n-1)}} \max |\phi|^{3}$ if and only if $M^{n}$ is totally umbilical.

Theorem 1.3. Let $M^{n}$ be an n-dimensional compact orientable space-like submanifold in an $(n+p)$-dimensional de Sitter space $S_{p}^{n+p}(1)$ with parallel normalized mean curvature vector field and let the scalar curvature $n(n-1) R$ be proportional to the mean curvature $H$ of $M^{n}$, that is, there exists a constant $k$ such that $n(n-1) R=k H$. Denote by $\lambda_{1}^{L_{R / H}}$ the first eigenvalue of the Schrödinger operator $L_{R / H}$. Then $\lambda_{1}^{L_{R / H}} \leq \frac{n-2}{\sqrt{n(n-1)}} \max |\phi|^{3}$, and $\lambda_{1}^{L_{R / H}}=\frac{n-2}{\sqrt{n(n-1)}} \max |\phi|^{3}$ if and only if $M^{n}$ is totally umbilical.

Corollary 1.4. Let $M^{n}$ be an $n$-dimensional compact space-like hypersurface in an $(n+1)$-dimensional de Sitter space $S_{1}^{n+1}(1)$ and let the scalar curvature $n(n-1) R$ be proportional to the mean curvature $H$ of $M^{n}$, that is, there exists a constant $k$ such that $n(n-1) R=k H$. Denote by $\lambda_{1}^{L_{R / H}}$ the first eigenvalue of the Schrödinger operator $L_{R / H}$. Then $\lambda_{1}^{L_{R / H}} \leq \frac{n-2}{\sqrt{n(n-1)}} \max |\phi|^{3}$, and $\lambda_{1}^{L_{R / H}}=\frac{n-2}{\sqrt{n(n-1)}} \max |\phi|^{3}$ if and only if $M^{n}$ is totally umbilical.

Remark 1.5. If $p=1$, then Theorem 1.1 and Theorem 1.2 reduce to Theorem 1 and Theorem 2 of [15], respectively. Therefore, we generalize the 
previous results obtained by [15] to general submanifolds with higher codimension.

\section{Preliminaries}

Let $S_{p}^{n+p}(1)$ be an $(n+p)$-dimensional de Sitter space with index $p$ and constant curvature 1 . Let $M^{n}$ be an $n$-dimensional connected space-like submanifold immersed in $S_{p}^{n+p}(1)$. We choose a local field of semi-Riemannian orthonormal frames $e_{1}, \ldots, e_{n+p}$ in $S_{p}^{n+p}(1)$ such that at each point of $M^{n}$, $e_{1}, \ldots, e_{n}$ span the tangent space of $M^{n}$ and form an orthonormal frame there. We use the following convention on the range of indices:

$$
1 \leq A, B, C, \ldots \leq n+p ; \quad 1 \leq i, j, k, \ldots \leq n, \quad n+1 \leq \alpha, \beta, \gamma, \ldots \leq n+p .
$$

Let $\omega_{1}, \ldots, \omega_{n+p}$ be its dual frame field so that the semi-Riemannian metric of $S_{p}^{n+p}(1)$ is given by $d \bar{s}^{2}=\sum_{i} \omega_{i}^{2}-\sum_{\alpha} \omega_{\alpha}^{2}=\sum_{A} \varepsilon_{A} \omega_{A}^{2}$, where $\varepsilon_{i}=1$ and $\varepsilon_{\alpha}=-1$. Then the structure equations of $S_{p}^{n+p}(1)$ are given by

$$
\begin{gathered}
d \omega_{A}=\sum_{B} \varepsilon_{B} \omega_{A B} \wedge \omega_{B}, \omega_{A B}+\omega_{B A}=0, \\
d \omega_{A B}=\sum_{C} \varepsilon_{C} \omega_{A C} \wedge \omega_{C B}-\frac{1}{2} \sum_{C, D} \varepsilon_{C} \varepsilon_{D} K_{A B C D} \omega_{C} \wedge \omega_{D}, \\
K_{A B C D}=\varepsilon_{A} \varepsilon_{B}\left(\delta_{A C} \delta_{B D}-\delta_{A D} \delta_{B C}\right) .
\end{gathered}
$$

If we restrict these form to $M^{n}$, then

$$
\omega_{\alpha}=0, \quad n+1 \leq \alpha \leq n+p
$$

From Cartan's Lemma we have

$$
\omega_{\alpha_{i}}=\sum_{j} h_{i j}^{\alpha} \omega_{j}, \quad h_{i j}^{\alpha}=h_{j i}^{\alpha} .
$$

The connection forms of $M^{n}$ are characterized by the structure equations

$$
\begin{gathered}
d \omega_{i}=\sum_{j=1}^{n} \omega_{i j} \wedge \omega_{j}, \quad \omega_{i j}+\omega_{j i}=0, \\
d \omega_{i j}=\sum_{k} \omega_{i k} \wedge \omega_{k j}-\frac{1}{2} \sum_{k, l} R_{i j k l} \omega_{k} \wedge \omega_{l},
\end{gathered}
$$




$$
R_{i j k l}=\left(\delta_{i k} \delta_{j l}-\delta_{i l} \delta_{j k}\right)-\sum_{\alpha}\left(h_{i k}^{\alpha} h_{j l}^{\alpha}-h_{i l}^{\alpha} h_{j k}^{\alpha}\right),
$$

where $R_{i j k l}$ are the components of the curvature tensor of $M^{n}$.

Denote by $h$ the second fundamental form of $M^{n}$. Then

$$
h=\sum_{i, j, \alpha} h_{i j}^{\alpha} \omega_{i} \otimes \omega_{j} \otimes e_{\alpha} .
$$

Denote by $\xi, H$ and $|h|^{2}$ the mean curvature vector field, the mean curvature and the squared norm of the second fundamental form of $M^{n}$. Then they are defined by

$$
\xi=\frac{1}{n} \sum_{\alpha}\left(\sum_{i} h_{i i}^{\alpha}\right) e_{\alpha}, H=|\xi|=\frac{1}{n} \sqrt{\sum_{\alpha}\left(\sum_{i} h_{i i}^{\alpha}\right)^{2}}, \quad|h|^{2}=\sum_{i, j, \alpha}\left(h_{i j}^{\alpha}\right)^{2} .
$$

Moreover, the normal curvature tensor $R_{\alpha \beta k l}$, the Ricci curvature tensor $R_{i k}$ and the scalar curvature $n(n-1) R$ are expressed as

$$
\begin{gathered}
R_{\alpha \beta k l}=\sum_{m}\left(h_{k m}^{\alpha} h_{m l}^{\beta}-h_{l m}^{\alpha} h_{m k}^{\beta}\right), \\
R_{i k}=(n-1) \delta_{i k}-\sum_{\alpha}\left(\sum_{l} h_{l l}^{\alpha}\right) h_{i k}^{\alpha}+\sum_{\alpha, j} h_{i j}^{\alpha} h_{j k}^{\alpha}, \\
n(n-1) R=n(n-1)+|h|^{2}-n^{2} H^{2},
\end{gathered}
$$

where $R$ is the normalized scalar curvature.

Define the first and the second covariant derivatives of $h_{i j}^{\alpha}$, say $h_{i j k}^{\alpha}$ and $h_{i j k l}^{\alpha}$ by

$$
\begin{gathered}
\sum_{k} h_{i j k}^{\alpha} \omega_{k}=d h_{i j}^{\alpha}+\sum_{k} h_{i k}^{\alpha} \omega_{k j}+\sum_{k} h_{j k}^{\alpha} \omega_{k i}-\sum_{\beta} h_{i j}^{\beta} \omega_{\beta \alpha} \\
\sum_{l} h_{i j k l}^{\alpha} \omega_{l}=d h_{i j k}^{\alpha}+\sum_{m} h_{m j k}^{\alpha} \omega_{m i}+\sum_{m} h_{i m k}^{\alpha} \omega_{m j}+\sum_{m} h_{i j m}^{\alpha} \omega_{m k}-\sum_{\beta} h_{i j k}^{\beta} \omega_{\beta \alpha} .
\end{gathered}
$$

We obtain the Codazzi equation by straightforward computations

$$
h_{i j k}^{\alpha}=h_{i k j}^{\alpha} \text {. }
$$

It follows that the Ricci identities hold

$$
h_{i j k l}^{\alpha}-h_{i j l k}^{\alpha}=\sum_{m} h_{i m}^{\alpha} R_{m j k l}+\sum_{m} h_{j m}^{\alpha} R_{m i k l}+\sum_{\beta} h_{i j}^{\beta} R_{\alpha \beta k l} .
$$


The Laplacian of $h_{i j}^{\alpha}$ is defined by $\Delta h_{i j}^{\alpha}=\sum_{k} h_{i j k k}^{\alpha}$. From (2.17) we obtain for any $\alpha, n+1 \leq \alpha \leq n+p$,

$$
\Delta h_{i j}^{\alpha}=\sum_{k} h_{k k i j}^{\alpha}+\sum_{k, m} h_{k m}^{\alpha} R_{m i j k}+\sum_{k, m} h_{i m}^{\alpha} R_{m k j k}+\sum_{k, \beta} h_{i k}^{\beta} R_{\alpha \beta j k} .
$$

In the case of the mean curvature vector $\xi$ has no zero, we know that $\xi / H$ is a normal vector field defined globally on $M^{n}$. We define $|\mu|^{2}$ and $|\tau|^{2}$ by

$$
|\mu|^{2}=\sum_{i, j}\left(h_{i j}^{n+1}-H \delta_{i j}\right)^{2}, \quad|\tau|^{2}=\sum_{\alpha>n+1} \sum_{i, j}\left(h_{i j}^{\alpha}\right)^{2},
$$

respectively. Then $|\mu|^{2}$ and $|\tau|^{2}$ are functions defined on $M^{n}$ globally, which do not depend on the choice of the orthonormal frame $\left\{e_{1}, \ldots, e_{n}\right\}$. We have

$$
|h|^{2}=n H^{2}+|\mu|^{2}+|\tau|^{2} .
$$

Since the normalized mean curvature vector field is parallel, we choose $e_{n+1}=$ $\xi / H$, then

$$
\operatorname{tr} H^{n+1}=\sum_{i} h_{i i}^{n+1}=n H, \quad \operatorname{tr} H^{\alpha}=\sum_{i} h_{i i}^{\alpha}=0 \quad(\alpha \geq n+2) .
$$

From (2.8), (2.11), (2.18) and (2.21), by a direct calculation we have (see [7])

$$
\begin{aligned}
\frac{1}{2} \Delta|h|^{2}= & \sum_{i, j, k, \alpha}\left(h_{i j k}^{\alpha}\right)^{2}+\sum_{i, j} h_{i j}^{n+1}(n H)_{i j}+n c\left(|h|^{2}-n H^{2}\right) \\
& -n H \sum_{\alpha} \operatorname{tr}\left(H_{\alpha}^{2} H_{n+1}\right)+\sum_{\alpha, \beta}\left[\operatorname{tr}\left(H_{\alpha} H_{\beta}\right)\right]^{2} \\
& +\sum_{\alpha, \beta} N\left(H_{\alpha} H_{\beta}-H_{\beta} H_{\alpha}\right),
\end{aligned}
$$

where $H_{\alpha}$ denote the matrix $\left(h_{i j}^{\alpha}\right)$ for all $\alpha, N(A)=\operatorname{tr}\left(A A^{t}\right)$, for all matrix $A=\left(a_{i j}\right)$.

We need the following Lemma

Lemma 2.1 ([13]). Let $A, B$ be symmetric $n \times n$ matrices satisfying $A B=B A$ and $\operatorname{tr} A=\operatorname{tr} B=0$. Then

$$
\left|\operatorname{tr} A^{2} B\right| \leq \frac{n-2}{\sqrt{n(n-1)}}\left(\operatorname{tr} A^{2}\right)\left(\operatorname{tr} B^{2}\right)^{1 / 2} .
$$

and the equality holds if and only if $(n-1)$ of the eigenvalues $x_{i}$ of $B$ and the corresponding eigenvalues $y_{i}$ of $A$ satisfy $\left|x_{i}\right|=\left(\operatorname{tr} B^{2}\right)^{1 / 2} / \sqrt{n(n-1)}$, 
$x_{i} x_{j} \geq 0, y_{i}=\left(\operatorname{tr} A^{2}\right)^{1 / 2} / \sqrt{n(n-1)}$.

We consider a differential operator $\square$ acting on function $f$ defined by

$$
\square f=\sum_{i, j=1}\left(n H \delta_{i j}-h_{i j}^{n+1}\right) f_{i j},
$$

where $d f=\sum_{i} f_{i} \omega_{i}, \quad \sum_{i, j} f_{i j} \omega_{j}=d f_{i}+\sum_{j} f_{j} \omega_{j i}$. From Cheng and Yau [9], we know that the operator $\square$ is self-adjoint if $M$ is compact.

We can prove that $\square$ is a elliptic operator. In fact, we choose a local orthonormal frame field $\left\{e_{1}, \ldots, e_{n}\right\}$ such that $h_{i j}^{n+1}=\lambda_{i} \delta_{i j}$. Since $R<1$, from (2.13), we have $|h|^{2}<n^{2} H^{2}$. If there is one $i$ such that $n H-\lambda_{i} \leq 0$, then $n^{2} H^{2} \leq \lambda_{i}^{2} \leq|h|^{2}$, this is a contradiction. Thus, we have $n H-\lambda_{i}>0$ for any $i$ and the operator $\square$ is elliptic.

From Proposition 3.6 in Section 3, we know that the differential operator $L=\square+(k / 2 n) \Delta$ is elliptic. We also know that the Laplace-Beltrami operator $\Delta$ is always elliptic. Let $\lambda_{1}^{L_{H}}, \lambda_{1}^{L_{R}}$ and $\lambda_{1}^{L_{R / H}}$ be the first eigenvalues of the Schrödinger operators $L_{H}, L_{R}$ and $L_{R / H}$ respectively. Since $\Delta, \square$ and $L$ are elliptic operators, from (1.1), (1.2) and (1.3), we know that $L_{H}, L_{R}$ and $L_{R / H}$ are elliptic operators. We can use the min-max characterization of $\lambda_{1}^{L_{H}}, \lambda_{1}^{L_{R}}$ and $\lambda_{1}^{L_{R / H}}$, as

$$
\begin{aligned}
& \lambda_{1}^{L_{H}}=\min \left\{\frac{\int_{M^{n}} f L_{H}(f) d v}{\int_{M^{n}} f^{2} d v} ; f \in \mathcal{C}^{\infty}\left(M^{n}\right), f \neq 0\right\}, \\
& \lambda_{1}^{L_{R}}=\min \left\{\frac{\int_{M^{n}} f L_{R}(f) d v}{\int_{M^{n}} f^{2} d v} ; f \in \mathcal{C}^{\infty}\left(M^{n}\right), f \neq 0\right\}, \\
& \lambda_{1}^{L_{R / H}}=\min \left\{\frac{\int_{M^{n}} f L_{R / H}(f) d v}{\int_{M^{n}} f^{2} d v} ; f \in \mathcal{C}^{\infty}\left(M^{n}\right), f \neq 0\right\} .
\end{aligned}
$$

\section{Proof of Theorems}

We firstly prove the following:

Proposition 3.1. Let $M^{n}$ be an $n$-dimensional space-like submanifold in an $(n+p)$-dimensional de Sitter space $S_{p}^{n+p}(1)$. Suppose that the normalized 
mean curvature vector field is parallel. Then

$$
\begin{aligned}
\square(n H) \geq & -\frac{1}{2} n(n-1) \Delta R+\left(|\nabla h|^{2}-n^{2}|\nabla H|^{2}\right) \\
& +|\phi|^{2}\left\{n-n H^{2}-\frac{n(n-2)}{\sqrt{n(n-1)}} H|\phi|+\frac{1}{p}|\phi|^{2}\right\} .
\end{aligned}
$$

Proof. By a simple calculation and from (2.13), we obtain

$$
\begin{aligned}
\square(n H) & =\sum_{i, j}\left(n H \delta_{i j}-h_{i j}^{n+1}\right)(n H)_{i j} \\
& =\frac{1}{2} \Delta\left(n^{2} H^{2}\right)-n^{2}|\nabla H|^{2}-\sum_{i, j} h_{i j}^{n+1}(n H)_{i j} \\
& =-\frac{1}{2} n(n-1) \Delta R+\frac{1}{2} \Delta|h|^{2}-n^{2}|\nabla H|^{2}-\sum_{i, j} h_{i j}^{n+1}(n H)_{i j} .
\end{aligned}
$$

Set $\phi_{i j}^{\alpha}=h_{i j}^{\alpha}-\frac{1}{n} \operatorname{tr} H^{\alpha} \delta_{i j}$ and consider the symmetric tensor $\phi=\sum_{i, j, \alpha} \phi_{i j}^{\alpha} \omega_{i} \omega_{j} e_{\alpha}$. We easy know that $\phi$ is traceless and

$$
N\left(\Phi_{\alpha}\right)=N\left(H_{\alpha}\right)-\frac{1}{n}\left(\operatorname{tr} H_{\alpha}\right)^{2}, \quad|\phi|^{2}=\sum_{\alpha} N\left(\Phi_{\alpha}\right)=|h|^{2}-n H^{2},
$$

where $\Phi_{\alpha}$ denotes the matrix $\left(\phi_{i j}^{\alpha}\right)$.

Since the normalized mean curvature vector field is parallel, choosing $e_{n+1}=\xi / H$, from $(2.21)$, we infer that

$$
\begin{aligned}
& \phi_{i j}^{n+1}=h_{i j}^{n+1}-H \delta_{i j}, \quad \phi_{i j}^{\alpha}=h_{i j}^{\alpha},(\alpha \geq n+2), \\
& N\left(\Phi_{n+1}\right)=N\left(H_{n+1}\right)-n H^{2}, \quad N\left(\Phi_{\alpha}\right)=N\left(H_{\alpha}\right),(\alpha \geq n+2), \\
& \operatorname{tr}\left(H_{n+1}\right)^{3}=\operatorname{tr}\left(\Phi_{n+1}\right)^{3}+3 H N\left(\Phi_{n+1}\right)+n H^{3} .
\end{aligned}
$$

From (2.22), (3.3) and (3.4), we have

$$
\begin{aligned}
\frac{1}{2} \Delta|h|^{2} \geq & \sum_{i, j, k, \alpha}\left(h_{i j k}^{\alpha}\right)^{2}+\sum_{i, j} h_{i j}^{n+1}(n H)_{i j}+n\left(c-H^{2}\right)|\phi|^{2} \\
& -n H \sum_{\alpha} \operatorname{tr}\left(\Phi_{\alpha}^{2} \Phi_{n+1}\right)+\sum_{\alpha, \beta}\left[\operatorname{tr}\left(\Phi_{\alpha} \Phi_{\beta}\right)\right]^{2} .
\end{aligned}
$$

Since we choose $e_{n+1}=\xi / H$, we have $\omega_{\alpha n+1}=0$ for all $\alpha$. Consequently $R_{\alpha n+1 j k}=0$, from (2.11), we have $\sum_{i} h_{i j}^{\alpha} h_{i k}^{n+1}=\sum_{i} h_{i k}^{\alpha} h_{i j}^{n+1}$, that is, 
$H_{\alpha} H_{n+1}=H_{n+1} H_{\alpha}$. Thus $\Phi_{\alpha} \Phi_{n+1}=\Phi_{n+1} \Phi_{\alpha}$. Since matrices $\Phi_{\alpha}$ and $\Phi_{n+1}$ are traceless, by Lemma 2.1 , we have

$$
\sum_{\alpha} \operatorname{tr}\left(\Phi_{\alpha}^{2} \Phi_{n+1}\right) \leq \frac{n-2}{\sqrt{n(n-1)}}|\mu||\phi|^{2} \leq \frac{n-2}{\sqrt{n(n-1)}}|\phi|^{3}
$$

where the following

$$
|\mu|^{2} \leq|h|^{2}-n H^{2}=|\phi|^{2},
$$

is used. By the Cauchy-Schwarz inequality, we have

$$
\sum_{\alpha, \beta}\left[\operatorname{tr}\left(\Phi_{\alpha} \Phi_{\beta}\right)\right]^{2} \geq \sum_{\alpha}\left[N\left(\Phi_{\alpha}\right)\right]^{2} \geq \frac{1}{p}|\phi|^{4}
$$

From (3.5), (3.6) and (3.8), we have

$$
\begin{aligned}
\frac{1}{2} \Delta|h|^{2} \geq & \sum_{i, j, k, \alpha}\left(h_{i j k}^{\alpha}\right)^{2}+\sum_{i, j} h_{i j}^{n+1}(n H)_{i j} \\
& +|\phi|^{2}\left\{n-n H^{2}-\frac{n(n-2)}{\sqrt{n(n-1)}} H|\phi|+\frac{1}{p}|\phi|^{2}\right\} .
\end{aligned}
$$

From (3.2) and (3.9), we see that (3.1) is true.

We state a Proposition which can be proved by making use of the similar method due to C. Wu [17] or A.A. Barros et al. [6] for Riemannian manifold.

Proposition 3.2. Let $M^{n}$ be an $n$-dimensional space-like submanifold in an $(n+p)$-dimensional de Sitter space $S_{p}^{n+p}(1)$. Then there holds the following

$$
\left.\left.|\nabla| \phi\right|^{2}\right|^{2} \leq \frac{4 n|\phi|^{2}}{n+2}|\nabla \phi|^{2}
$$

From the author [14], we also have the following:

Proposition 3.3. Let $M^{n}$ be an $n$-dimensional space-like submanifold in an $(n+p)$-dimensional de Sitter space $S_{p}^{n+p}(1)$. Suppose that the normalized scalar curvature $R$ is constant and $R \leq 1$. Then

$$
|\nabla h|^{2} \geq n^{2}|\nabla H|^{2}
$$

Remark 3.4. If $|\nabla h|^{2}=n^{2}|\nabla H|^{2}$ and $R<1$, from (2.13) and the proof of Proposition 3.3 (see [14]), we easily see that

$$
0 \leq n^{3}(n-1)(1-R)|\nabla H|^{2} \leq S\left(|\nabla h|^{2}-n^{2}|\nabla H|^{2}\right) .
$$


Thus, we have $\nabla H=0$ and $H$ is constant.

Proof of theorem 1.1 From the definition of (2.10), we know that $H \geq 0$. For every $\varepsilon>0$, from (2.25), we can introduce a smooth function $f_{\varepsilon}=\sqrt{\varepsilon+|\phi|^{2}}$ as the test function to estimate $\lambda_{1}^{L_{H}}$. We easily have

$$
\Delta f_{\varepsilon}=\frac{1}{2 \sqrt{\varepsilon+|\phi|^{2}}} \Delta|\phi|^{2}-\left.\left.\frac{1}{4\left(\varepsilon+|\phi|^{2}\right)^{3 / 2}}|\nabla| \phi\right|^{2}\right|^{2} .
$$

Since we know that submanifolds with nonzero parallel mean curvature vector field also have parallel normalized mean curvature vector field and $H$ is nonzero constant, from (3.9), we easily see that

$$
\frac{1}{2} \Delta|\phi|^{2} \geq|\phi|^{2}\left\{n-n H^{2}-\frac{n(n-2)}{\sqrt{n(n-1)}} H|\phi|+\frac{1}{p}|\phi|^{2}\right\} .
$$

By Proposition 3.2, (3.12) and (3.13), we have

$$
\begin{aligned}
f_{\varepsilon} \Delta f_{\varepsilon}= & \frac{1}{2} \Delta|\phi|^{2}-\left.\left.\frac{1}{4\left(\varepsilon+|\phi|^{2}\right)}|\nabla| \phi\right|^{2}\right|^{2} \\
\geq & |\phi|^{2}\left\{\frac{1}{p}|\phi|^{2}-\frac{n(n-2) H}{\sqrt{n(n-1)}}|\phi|+n\left(1-H^{2}\right)\right\} \\
& +|\nabla \phi|^{2}-\left.\left.\frac{1}{4\left(\varepsilon+|\phi|^{2}\right)}|\nabla| \phi\right|^{2}\right|^{2} \\
\geq & |\phi|^{2}\left\{\frac{1}{p}|\phi|^{2}-\frac{n(n-2) H}{\sqrt{n(n-1)}}|\phi|+n\left(1-H^{2}\right)\right\} \\
& +\left\{1-\frac{n|\phi|^{2}}{(n+2)\left(\varepsilon+|\phi|^{2}\right)}\right\}|\nabla \phi|^{2} .
\end{aligned}
$$

Therefore, we have

$$
\begin{aligned}
f_{\varepsilon} L_{H} f_{\varepsilon}= & -f_{\varepsilon} \Delta f_{\varepsilon}+\left\{\frac{1}{p}|\phi|^{2}-\frac{n(n-2)}{\sqrt{n(n-1)}} H|\phi|\right\} f_{\varepsilon}^{2} \\
\leq & -|\phi|^{2}\left\{\frac{1}{p}|\phi|^{2}-\frac{n(n-2) H}{\sqrt{n(n-1)}}|\phi|+n\left(1-H^{2}\right)\right\} \\
& -\left\{1-\frac{n|\phi|^{2}}{(n+2)\left(\varepsilon+|\phi|^{2}\right)}\right\}|\nabla \phi|^{2} \\
& +\left\{\frac{1}{p}|\phi|^{2}-\frac{n(n-2)}{\sqrt{n(n-1)}} H|\phi|\right\}\left(\varepsilon+|\phi|^{2}\right) .
\end{aligned}
$$


Using (2.25) with $f_{\varepsilon}$ as a test function, we have

$$
\begin{aligned}
\lambda_{1}^{L_{H}} \int_{M^{n}}\left(\varepsilon+|\phi|^{2}\right) d v=\lambda_{1}^{L_{H}} \int_{M^{n}} f_{\varepsilon}^{2} d v \leq \int_{M^{n}} f_{\varepsilon} L_{H}\left(f_{\varepsilon}\right) d v \\
\leq-\int_{M^{n}}|\phi|^{2}\left\{\frac{1}{p}|\phi|^{2}-\frac{n(n-2) H}{\sqrt{n(n-1)}}|\phi|+n\left(1-H^{2}\right)\right\} d v \\
-\int_{M^{n}}\left\{1-\frac{n|\phi|^{2}}{(n+2)\left(\varepsilon+|\phi|^{2}\right)}\right\}|\nabla \phi|^{2} d v \\
+\int_{M^{n}}\left\{\frac{1}{p}|\phi|^{2}-\frac{n(n-2)}{\sqrt{n(n-1)}} H|\phi|\right\}\left(\varepsilon+|\phi|^{2}\right) d v .
\end{aligned}
$$

Letting $\varepsilon \rightarrow \infty$ in (3.14), we get

$$
\lambda_{1}^{L_{H}} \int_{M^{n}}|\phi|^{2} d v \leq-n\left(1-H^{2}\right) \int_{M^{n}}|\phi|^{2} d v-\int_{M^{n}} \frac{2}{n+2}|\nabla \phi|^{2} d v .
$$

Since $\lambda_{1}^{L_{H}} \geq-n\left(1-H^{2}\right)$, from $(3.15)$, we have $|\nabla \phi|^{2}=0$. Proposition 3.2 implies that $\nabla|\phi|^{2}=0$, that is, $|\phi|^{2}$ is constant. Therefore, we know that $\frac{1}{p}|\phi|^{2}-\frac{n(n-2)}{\sqrt{n(n-1)}} H|\phi|$ is constant. From (1.1), we obtain that $\lambda_{1}^{L_{H}}=$ $\frac{1}{p}|\phi|^{2}-\frac{n(n-2)}{\sqrt{n(n-1)}} H|\phi|$. So we have

$$
-n\left(1-H^{2}\right) \leq \frac{1}{p}|\phi|^{2}-\frac{n(n-2)}{\sqrt{n(n-1)}} H|\phi|,
$$

that is

$$
\frac{1}{p}|\phi|^{2}-\frac{n(n-2)}{\sqrt{n(n-1)}} H|\phi|+n\left(1-H^{2}\right) \geq 0 .
$$

Therefore, we know that the equalities in (3.13), (3.8), (3.7) and (2.23) hold and

$$
|\phi|^{2}\left\{\frac{1}{p}|\phi|^{2}-\frac{n(n-2)}{\sqrt{n(n-1)}} H|\phi|+n\left(1-H^{2}\right)\right\}=0 .
$$

This implies that $|\phi|^{2}=0$, that is, $M^{n}$ is totally umbilical, or

$$
\frac{1}{p}|\phi|^{2}-\frac{n(n-2)}{\sqrt{n(n-1)}} H|\phi|+n\left(1-H^{2}\right)=0 .
$$

In the second case, from the equalities in (3.8) and (3.7), we have $|\mu|^{2}=|\phi|^{2}$ 
and $p \sum_{\alpha, \beta}\left[\operatorname{tr}\left(\Phi_{\alpha} \Phi_{\beta}\right)\right]^{2}=|\phi|^{4}$. Thus, we have

$$
\begin{aligned}
|\phi|^{4}= & p\left\{\left(\operatorname{tr} \Phi_{n+1}^{2}\right)^{2}+\sum_{\alpha>n+1}\left[\operatorname{tr}\left(\Phi_{\alpha} \Phi_{n+1}\right)\right]^{2}\right. \\
& \left.+\sum_{\beta>n+1}\left[\operatorname{tr}\left(\Phi_{n+1} \Phi_{\beta}\right)\right]^{2}+\sum_{\alpha, \beta>n+1}\left[\operatorname{tr}\left(\Phi_{\alpha} \Phi_{\beta}\right)\right]^{2}\right\}
\end{aligned}
$$

From (3.4), we have

$$
\begin{aligned}
(p-1)|\phi|^{4} & +p \sum_{\alpha>n+1}\left[\operatorname{tr}\left(\Phi_{\alpha} \Phi_{n+1}\right)\right]^{2} \\
& +p \sum_{\beta>n+1}\left[\operatorname{tr}\left(\Phi_{n+1} \Phi_{\beta}\right)\right]^{2}+p \sum_{\alpha, \beta>n+1}\left[\operatorname{tr}\left(\Phi_{\alpha} \Phi_{\beta}\right)\right]^{2}=0
\end{aligned}
$$

Since $p \geq 1$ and all parts of the above equality are nonnegative, we have $(p-1)|\phi|^{4}=0$. Thus, $|\phi|^{2}=0$ and $M^{n}$ is totally umbilical, or $p=1$. If $p=1$, we know that $M^{n}$ is an $n$-dimensional compact orientable space-like hypersurface in an $(n+1)$-dimensional de Sitter space $S_{1}^{n+1}(1)$ with constant mean curvature $H$. From the equalities in (2.23), we infer that $M^{n}$ has at most two distinct constant principal curvatures. We conclude that $M^{n}$ is totally umbilical from the compactness of $M^{n}$. This completes the proof of Theorem 1.1.

Proof of theorem $\mathbf{1 . 2}$ Since $n(n-1) R$ is constant and $R<1$, by Proposition 3.1 and Proposition 3.3, we have

$$
\square(n H) \geq|\phi|^{2}\left\{n-n H^{2}-\frac{n(n-2)}{\sqrt{n(n-1)}} H|\phi|+\frac{1}{p}|\phi|^{2}\right\} .
$$

From the assertion in Section 2, we know that the operator $\square$ is elliptic. Since we assume that the normalized mean curvature vector $e_{n+1}=\xi / H$ is parallel, we have $H>0$. Thus, from (2.26), we introduce a smooth function $f=H$ as the test function to estimate $\lambda_{1}^{L_{R}}$. By (1.2) and (3.16), we have

$$
\begin{aligned}
L_{R}(H)= & -\square(H)+\frac{1}{n p}|\phi|^{4}+\left(1-H^{2}\right)|\phi|^{2} \\
\leq & -\frac{1}{n p}|\phi|^{4}-\left(1-H^{2}\right)|\phi|^{2}+\frac{n-2}{\sqrt{n(n-1)}} H|\phi|^{3} \\
& +\frac{1}{n p}|\phi|^{4}+\left(1-H^{2}\right)|\phi|^{2}=\frac{n-2}{\sqrt{n(n-1)}} H|\phi|^{3} .
\end{aligned}
$$


From (2.26) and (3.17), we have

$$
\begin{aligned}
\lambda_{1}^{L_{R}} \int_{M^{n}} H^{2} d v & \leq \int_{M^{n}} H L_{R}(H) d v \\
& \leq \int_{M^{n}} \frac{n-2}{\sqrt{n(n-1)}} H^{2}|\phi|^{3} d v \\
& \leq \frac{n-2}{\sqrt{n(n-1)}} \max |\phi|^{3} \int_{M^{n}} H^{2} d v
\end{aligned}
$$

Thus, we have

$$
\lambda_{1}^{L_{R}} \leq \frac{n-2}{\sqrt{n(n-1)}} \max |\phi|^{3} .
$$

If $\lambda_{1}^{L_{R}}=\frac{n-2}{\sqrt{n(n-1)}} \max |\phi|^{3}$, then the equalities in (3.18), (3.17), (3.16), (3.11), (3.8), (3.7) and (2.23) hold. Since the operator $\square$ is self-adjoint and $M^{n}$ is compact, from the equality of (3.16), we obtain that

$$
\int_{M^{n}}|\phi|^{2}\left\{\frac{1}{p}|\phi|^{2}-\frac{n(n-2) H}{\sqrt{n(n-1)}}|\phi|+n\left(1-H^{2}\right)\right\} d v=0 .
$$

This implies that $|\phi|^{2}=0$ and $M^{n}$ is totally umbilical, or

$$
\frac{1}{p}|\phi|^{2}-\frac{n(n-2) H}{\sqrt{n(n-1)}}|\phi|+n\left(1-H^{2}\right)=0 .
$$

In the second case, since $R<1$ and the equality holds in (3.11), from the Remark 3.4, we know that $H$ is constant. From the equalities of (3.8), (3.7) and (2.23), by the same assertion in the proof of Theorem 1.1, we know that $M^{n}$ is an $n$-dimensional compact orientable space-like hypersurface in de Sitter space $S_{1}^{n+1}(1)$ with at most two distinct constant principal curvatures. We conclude that $M^{n}$ is totally umbilical from the compactness of $M^{n}$. If $M^{n}$ is totally umbilical, that is $|\phi|=0$, from (1.2), we know that $\lambda_{1}^{L_{R}}=\frac{1}{n p H}|\phi|^{4}+$ $\frac{1-H^{2}}{H}|\phi|^{2}=0$. This completes the proof of Theorem 1.2.

We may also prove the following:

Proposition 3.5. Let $M^{n}$ be an n-dimensional space-like submanifold in an $(n+p)$-dimensional de Sitter space $S_{p}^{n+p}(1)$. If the scalar curvature $n(n-1) R$ is proportional to the mean curvature $H$ of $M^{n}$, that is, there exists a constant $k$ such that $n(n-1) R=k H$, then we have

$$
|\nabla h|^{2} \geq n^{2}|\nabla H|^{2}
$$


In particular, if $|\nabla h|^{2}=n^{2}|\nabla H|^{2}$, then $H$ is constant.

Proof. For a fixed $\alpha$, we choose a orthonormal frame field $\left\{e_{i}\right\}$ at each point on $M^{n}$ so that $h_{i j}^{\alpha}=\lambda_{i}^{\alpha} \delta_{i j}$. Then we have $|h|^{2}=\sum_{i, j, \alpha}\left(h_{i j}^{\alpha}\right)^{2} \neq 0$. In fact, if $|h|^{2}=\sum_{i, \alpha}\left(\lambda_{i}^{\alpha}\right)^{2}=0$ at a point of $M^{n}$, then $\lambda_{i}^{\alpha}=0$ for all $i$ and $\alpha$ at this point. This implies $H=0$ and $R=0$ at this point. From (2.13), we have $n(n-1)=0$. This is impossible. From $(2.13)$ and $n(n-1) R=k H$, we have

$$
\begin{gathered}
k \nabla_{i} H=-2 n^{2} H \nabla_{i} H+2 \sum_{j, k, \alpha} h_{k j}^{\alpha} h_{k j i}^{\alpha}, \\
\left(\frac{k}{2}+n^{2} H\right)^{2}|\nabla H|^{2}=\sum_{i}\left(\sum_{j, k, \alpha} h_{k j}^{\alpha} h_{k j i}^{\alpha}\right)^{2} \leq \sum_{i, j, \alpha}\left(h_{i j}^{\alpha}\right)^{2} \sum_{i, j, k, \alpha}\left(h_{i j k}^{\alpha}\right)^{2}=|h|^{2}|\nabla h|^{2} .
\end{gathered}
$$

Thus, we have

$$
\begin{aligned}
|\nabla h|^{2}-n^{2}|\nabla H|^{2} & \geq\left[\left(\frac{k}{2}+n^{2} H\right)^{2}-n^{2}|h|^{2}\right]|\nabla H|^{2} \frac{1}{|h|^{2}} \\
& =\left[\frac{(k)^{2}}{4}+n^{3}(n-1)\right]|\nabla H|^{2} \frac{1}{|h|^{2}} \geq 0 .
\end{aligned}
$$

If $|\nabla h|^{2}=n^{2}|\nabla H|^{2}$, we easily see that $\nabla H=0$ and $H$ is constant. The proof of Proposition 3.5 is completed.

Proposition 3.6. Let $M^{n}$ be an $n$-dimensional space-like submanifold in a de Sitter space $S_{p}^{n+p}(1)$. If $n(n-1) R=k H$ and $H>0$, then the differential operator $L=\square+(k / 2 n) \Delta$ is elliptic.

Proof. For a fixed $\alpha$, we choose a local orthonormal frame field $\left\{e_{1}, \ldots, e_{n}\right\}$ at each point on $M^{n}$ so that $h_{i j}^{\alpha}=\lambda_{i}^{\alpha} \delta_{i j}$. From $H>0, n H=\sum_{i} h_{i i}^{n+1}$ and $\sum_{i} h_{i i}^{\alpha}=0$ for $n+2 \leq \alpha \leq n+p$ on $M^{n}$, we have, for any $i$

$$
\begin{aligned}
& \left(n H-\lambda_{i}^{n+1}+k / 2 n\right)=\sum_{j} \lambda_{j}^{n+1}-\lambda_{i}^{n+1} \\
& \quad+(1 / 2)\left[\sum_{j, \alpha}\left(\lambda_{j}^{\alpha}\right)^{2}-n^{2} H^{2}+n(n-1)\right] /(n H) \\
& \geq \sum_{j} \lambda_{j}^{n+1}-\lambda_{i}^{n+1} \\
& \quad+(1 / 2)\left[\sum_{j}\left(\lambda_{j}^{n+1}\right)^{2}-\left(\sum_{j} \lambda_{j}^{n+1}\right)^{2}+n(n-1)\right] /(n H)
\end{aligned}
$$




$$
\begin{aligned}
= & {\left[\left(\sum_{j} \lambda_{j}^{n+1}\right)^{2}-\lambda_{i}^{n+1}\left(\sum_{j} \lambda_{j}^{n+1}\right)\right.} \\
& \left.-(1 / 2) \sum_{l \neq j} \lambda_{l}^{n+1} \lambda_{j}^{n+1}+(1 / 2) n(n-1)\right](n H)^{-1} \\
= & {\left[\sum_{j}\left(\lambda_{j}^{n+1}\right)^{2}+(1 / 2) \sum_{l \neq j} \lambda_{l}^{n+1} \lambda_{j}^{n+1}\right.} \\
& \left.-\lambda_{i}^{n+1}\left(\sum_{j} \lambda_{j}^{n+1}\right)+(1 / 2) n(n-1)\right](n H)^{-1} \\
= & {\left[\sum_{j \neq i}\left(\lambda_{j}^{n+1}\right)^{2}+(1 / 2) \sum_{\substack{l \neq j \\
l, j \neq i}} \lambda_{l}^{n+1} \lambda_{j}^{n+1}+(1 / 2) n(n-1)\right](n H)^{-1} } \\
= & (1 / 2)\left[\sum_{j \neq i}\left(\lambda_{j}^{n+1}\right)^{2}+\left(\sum_{j \neq i} \lambda_{j}^{n+1}\right)^{2}+n(n-1)\right](n H)^{-1}>0 .
\end{aligned}
$$

Thus, $L$ is an elliptic operator. The proof of Lemma 3.6 is completed.

Proof of theorem 1.3 Since $n(n-1) R=k H$, by Proposition 3.1 and Proposition 3.5, we have

$$
L(n H) \geq|\phi|^{2}\left\{n-n H^{2}-\frac{n(n-2)}{\sqrt{n(n-1)}} H|\phi|+\frac{1}{p}|\phi|^{2}\right\} .
$$

From Proposition 3.6, we know that the operator $L$ is elliptic. Since we assume that the normalized mean curvature vector $e_{n+1}=\xi / H$ is parallel, we have $H>0$. Thus, from (2.27), we introduce a smooth function $f=H$ as the test function to estimate $\lambda_{1}^{L_{R / H}}$. By (1.3) and (3.21), we have

$$
\begin{aligned}
L_{R / H}(H)= & -L(H)+\frac{1}{n p}|\phi|^{4}+\left(1-H^{2}\right)|\phi|^{2} \\
\leq & -\frac{1}{n p}|\phi|^{4}-\left(1-H^{2}\right)|\phi|^{2}+\frac{n-2}{\sqrt{n(n-1)}} H|\phi|^{3} \\
& +\frac{1}{n p}|\phi|^{4}+\left(1-H^{2}\right)|\phi|^{2}=\frac{n-2}{\sqrt{n(n-1)}} H|\phi|^{3} .
\end{aligned}
$$

Thus

$$
\begin{aligned}
\lambda_{1}^{L_{R / H}} \int_{M^{n}} H^{2} d v & \leq \int_{M^{n}} H L_{R / H}(H) d v \\
& \leq \int_{M^{n}} \frac{n-2}{\sqrt{n(n-1)}} H^{2}|\phi|^{3} d v \\
& \leq \frac{n-2}{\sqrt{n(n-1)}} \max |\phi|^{3} \int_{M^{n}} H^{2} d v .
\end{aligned}
$$


We have $\lambda_{1}^{L_{R / H}} \leq \frac{n-2}{\sqrt{n(n-1)}} \max |\phi|^{3}$. If $\lambda_{1}^{L_{R / H}}=\frac{n-2}{\sqrt{n(n-1)}} \max |\phi|^{3}$, then the equalities in (3.23), (3.22), (3.21), (3.20), (3.8), (3.7) and (2.23) hold. By the same method of the proof of Theorem 1.2, we know that if and only if $M^{n}$ is totally umbilical. This completes the proof of Theorem 1.3.

Acknowledgment. Project supported by NSF of Shaanxi Educational Department (2010JK532, 11JK0479) and NSF of Weinan Normal University (2012YKS026).

\section{References}

[1] K. Akutagawa, On space-like hypersurfaces with constant mean curvature in a de Sitter space, Math. Z., 196 (1987), 13-19.

[2] H. Alencar, M. do Carmo and A.G. Colares, Stable hypersurfaces with constant scalar curvature, Math. Z., 213 (1993), 117-131.

[3] J.L. Barbosa, M. do Carmo and J. Eschenburg, Stability of hypersurfaces of constant mean curvature in Riemannian manifolds, Math. Z., 197 (1988), 123-138.

[4] J.L. Barbosa and V. Oliker, Stable space-like hypersurfaces with constant mean curvature in Lorentz space, Geometry and global Analysis, Tohoku Univ. Sendai, (1993), 161-164.

[5] J.L. Barbosa and V. Oliker, Space-like hypersurfaces with constant mean curvature in Lorentz space, Mat. Contemp, 4 (1993), 27-44.

[6] A.A. Barros, A.C. Brasil Jr. and L.A.M. Sousa Jr., A new characterization of submanifolds with parallel mean curvature vector in $S^{n+p}$, Kodai Math. J., 27 (2004), 45-56.

[7] F.E. C Camargo and R.S. B. Chaves and L.A. M. De Sousa Jr, New characterizations of complete spacelike submanifolds in semi-Riemannian space forms, Kodai Math. J., 32 (2009), 209-230.

[8] Q.-M. Cheng, First eigenvalue of a Jacobi operator of hypersurfaces with a constant scalar curvature, Proc. Amer. Math. Soc., 136 (2008), 33093318 .

[9] S.Y. Cheng and S.T. Yau, Hypersurfaces with constant scalar curvature, Math. Ann., 225 (1977), 195-204. 
[10] X. Liu and J. Deng, Stable space-like hypersurfaces in the de Sitter space, Archivum Math. (Brno), 40 (2004), 111-117.

[11] J. Marsden and F. Tipler, Maximal hypersurfaces and foliations of constant mean curvature in general relativity, Bull. Am. phys. Soc., 23 (1978), 84-90.

[12] S. Montiel, A characterization of hyperbolic cylinders in the de Sitter space, Tôhoku Math. J., 48 (1996), 23-31.

[13] W. Santos, Submanifolds with parallel mean curvature vector in spheres, Tôhoku Math. J, 46 (1994), 403-415.

[14] S. Shu and S. Liu, Complete space-like submanifolds with constant scalar curvature in a de Sitter space, Balkan J. of Geom. Appli., 9 (2004), 82-91.

[15] S. Shu and S. Liu, First eigenvalue of Schrödinger operator of space-like hypersurfaces, Anziam J., 51 (2010), 83-96.

[16] S. Stumbles, Hypersurfaces of constant mean extrinsic curvature, Ann. Phys., 133 (1980), 28-56

[17] C. Wu, New characterizations of the Clifford tori and the Veronese surface, Arch. Math., 61 (1993) 277-284.

Shichang SHU,

School of Mathematics and Information Science,

Xianyang Normal University,

Xianyang, 712000, Shaanxi, P.R. China.

Email: shushichang@126.com

Tianmin ZHU,

College of Mathematics and Information Science,

Weinan Normal University,

Weinan, 714000, Shaanxi, P.R. China.

Email: wnzhutm@163.com 